\title{
Prognostic analysis of uterine cervical cancer treated with postoperative radiotherapy: importance of positive or close parametrial resection margin
}

\author{
Yi-Jun Kim, MD¹, Kyung-Ja Lee, MD, PhD1, Kyung Ran Park, MD, PhD', Jiyoung Kim, MD¹, \\ Wonguen Jung, MD', Rena Lee, $\mathrm{PhD}^{1}$, Seung Cheol Kim, MD, PhD², Hye Sung Moon, MD, PhD², \\ Woong Ju, MD, PhD², Yun Hwan Kim, MD, PhD², Jihae Lee, MD \\ Departments of ${ }^{1}$ Radiation Oncology and ${ }^{2}$ Obstetrics and Gynecology, Ewha Womans University Mokdong Hospital, \\ Ewha Womans University School of Medicine, Seoul, Korea
}

Purpose: To analyze prognostic factors for locoregional recurrence (LRR), distant metastasis (DM), and overall survival (OS) in cervical cancer patients who underwent radical hysterectomy followed by postoperative radiotherapy (PORT) in a single institute. Materials and Methods: Clinicopathologic data of 135 patients with clinical stage IA2 to IIA2 cervical cancer treated with PORT from 2001 to 2012 were reviewed, retrospectively. Postoperative parametrial resection margin (PRM) and vaginal resection margin (VRM) were investigated separately. The median treatment dosage of external beam radiotherapy (EBRT) to the whole pelvis was $50.4 \mathrm{~Gy}$ in $1.8 \mathrm{~Gy} /$ fraction. High-dose-rate vaginal brachytherapy after EBRT was given to patients with positive or close VRMs. Concurrent platinum-based chemoradiotherapy (CCRT) was administered to 73 patients with positive resection margin, lymph node (LN) metastasis, or direct extension of parametrium. Kaplan-Meier method and log-rank test were used for analyzing LRR, DM, and OS; Cox regression was applied to analyze prognostic factors.

Results: The 5-year disease-free survival was 79\% and 5-year OS was 91\%. In univariate analysis, positive or close PRM, LN metastasis, direct extension of parametrium, lymphovascular invasion, histology of adenocarcinoma, and chemotherapy were related with more DM and poor OS. In multivariate analysis, PRM and LN metastasis remained independent prognostic factors for OS.

Conclusion: PORT after radical hysterectomy in uterine cervical cancer showed excellent OS in this study. Positive or close PRM after radical hysterectomy in uterine cervical cancer correlates with poor prognosis even with CCRT. Therefore, additional treatments to improve local control such as radiation boosting need to be considered.

Keywords: Parametrial resection margin, Uterine cervical neoplasms, Postoperative adjuvant radiotherapy

\section{Introduction}

Treatment options for uterine cervical cancer include surgery, radiotherapy (RT) and chemotherapy; among these, hysterectomy with regional lymph node (LN) dissection has been established as a primary treatment option in cervical cancer FIGO stage IB1IIA [1]. Adding postoperative radiotherapy (PORT) at a total dose of 46-50.4 Gy to the whole pelvis improves survival outcomes

Received 6 April 2015, Revised 17 May 2015, Accepted 1 June 2015.

Correspondence: Jihae Lee, MD, Department of Radiation Oncology, Ewha Womans University Mokdong Hospital, 1071 Anyangcheon-ro, Yangcheon-gu, Seoul 158-710, Korea. Tel: +82-2-2650-5331, Fax: +82-2-2654-0363, E-mail: jihae.leee@ gmail.com

(c) This is an Open Access article distributed under the terms of the Creative Commons Attribution Non-Commercial License (http://creativecommons.org/ licenses/by-nc/4.0/) which permits unrestricted non-commercial use, distribution, and reproduction in any medium, provided the original work is properly cited.

www.e-roj.org 
[2]. Addition of chemotherapy to pelvic RT is indicated if a

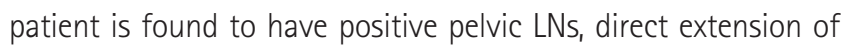
parametrium, or positive surgical margin after hysterectomy and chemotherapy improves prognosis of these high-risk cervical cancer patients [3].

Concurrent chemoradiotherapy (CCRT) decreased both the rate of local failure and distant failure as the chemotherapy acts as a radiation sensitizer [4]. But unlike resected metastatic LNs and direct extension of parametrium with clear margin after hysterectomy with pelvic lymph nodal dissection, positive resection margin (RM) signifies still remnant tumor tissues in operation bed suggesting more aggressive treatment for local control may be considered.

According to the National Comprehensive Cancer Network (NCCN) guidelines [5], adjuvant CCRT for uterine cervical cancer with a positive RM is recommended. If the vaginal resection margin (VRM) is positive, additional vaginal brachytherapy with a dose range of 10-15 Gy is an option, whereas positive parametrial resection margin (PRM) is not a standard indication for radiation boosting. The Gynecologic Oncology Group (GOG) 109 clinical trial showed that no vaginal brachytherapy was indicated for tumors with positive RMs since the study established the improved prognosis associated with postoperative chemotherapy for high-risk cervical cancer [3]. However, since subanalysis for patients with positive surgical margins has not been conducted, it is not clear whether adding chemotherapy to conventional pelvic $\mathrm{RT}$ is enough to compensate for the poor prognosis of positive RMs.

The aim of the present study was to analyze the prognostic significance of risk factors in the era of postoperative adjuvant CCRT for the treatment of cervical cancer. For RM analysis, PRM and VRM data were divided.

\section{Materials and Methods}

Between 2001 and 2012, 142 women with clinical stages IA2 to IIA2 cervical cancer patients were treated with radical hysterectomy and PORT with or without chemotherapy in the Department of Radiation Oncology in a single institute. Surgical procedures included radical hysterectomy and bilateral salpingo-oophorectomy. Pelvic LN dissection was performed on 121 patients (90\%). Para-aortic LN sampling was performed in 37 cases with suspicion of metastases. The median number of pelvic LNs removed was 36 (range, 12 to 88). Patients who had two or more of the following risk factors were indicated for PORT without concurrent chemotherapy:
Iymphovascular invasion, depth of invasion to $1 / 2$ or more of cervix stroma, and bulky tumor size. Postoperative CCRT was prescribed to patients with the following pathologic risk factors: LN metastasis, direct extension of parametrium, or positive RM. Positive RMs include surgical positive PRM (pllB) and positive VRM. Close RM is defined as RM within $1 \mathrm{~mm}$. The patient characteristics are described in Table 1.

Pelvic EBRT was delivered with $10 \mathrm{MV} X$-rays and mainly by a four-field box technique. The daily dose was $1.8 \mathrm{~Gy}$, 5 fractions per week. The treatment field was categorized as extended fields (para-aortic region plus whole pelvis) or whole pelvis on the basis of the level of LN metastasis. Among all patients, 2 patients had pathologic positive paraaortic LNs and including these 2 patients, a total of 8 patients who had clinically suspicious metastatic para-aortic LNs on computed tomography (CT) or magnetic resonance imaging (MRI) received extended-field RT; 127 patients received whole pelvic irradiation only. The treatment dosage to whole pelvis consisted of 45.0-54.0 Gy and majority were treated with 50.4 Gy (91\%). Treatment dose to the para-aortic region was 45.0 Gy.

Of the 135 patients, 6 patients had positive or close PRMs, 6 patients had positive or close VRMs, and one patient had both PRM and VRM involvements. All 7 patients with positive or close PRMs had direct extensions of parametrium (pllB) and including these 7 patients, total 28 patients had direct extensions of parametrium. High-dose-rate (HDR) vaginal cuff brachytherapy after EBRT was given to 5 of 7 patients with positive or close VRMs and 2 of 7 patients with positive or close PRMs. Vaginal brachytherapy was performed after 4,140 cGy of pelvic irradiation and additional EBRT was delivered with a midline block. HDR vaginal brachytherapy was performed using an iridium-192 source. The proximal $3-4 \mathrm{~cm}$ of vaginal cuff was treated using vaginal ovoids. Brachytherapy was given in five to six fractions with 3-4 Gy at $5 \mathrm{~mm}$ depth of vaginal cuff per fraction, twice a week.

Adjuvant RT alone was given to 62 patients (46\%), whereas the remaining 73 patients received CCRT (54\%). Specific chemotherapeutic regimen decisions were made according to the preference of the responsible surgeon. For those treated with chemotherapy, 30 patients received weekly cisplatin $\left(40 \mathrm{mg} / \mathrm{m}^{2}\right)$ and another 34 patients received platinumbased combination regimens. Neoadjuvant cisplatin and 5-fluorouracil was administered to 9 patients.

After completion of PORT or CCRT, each patient underwent regular follow-up every 3 months in the first year, then every 6 months subsequently. A pelvic examination was performed 
Table 1. Univariate analysis of LRR, DM, OS by clinicopathological characteristics

\begin{tabular}{|c|c|c|c|c|}
\hline \multirow{2}{*}{ Clinicopathological factor } & \multirow{2}{*}{$\begin{array}{c}\text { No. of } \\
\text { patients }\end{array}$} & \multicolumn{3}{|c|}{$p$-value } \\
\hline & & LRR & DM & OS \\
\hline Age (yr) & & 0.172 & 0.997 & 0.814 \\
\hline$<40$ & 21 & & & \\
\hline$\geq 40$ & 114 & & & \\
\hline FIGO stage & & 0.914 & 0.354 & 0.051 \\
\hline IA2 & 2 & & & \\
\hline IB1 & 80 & & & \\
\hline IB2 & 14 & & & \\
\hline$\| \mathrm{A} 1$ & 28 & & & \\
\hline$\| \mathrm{A} 2$ & 11 & & & \\
\hline Tumor size (cm) & & 0.488 & 0.120 & 0.258 \\
\hline$\leq 4$ & 102 & & & \\
\hline$>4$ & 26 & & & \\
\hline Null & 7 & & & \\
\hline Lymphovascular invasion & & 0.029 & 0.000 & 0.005 \\
\hline No & 65 & & & \\
\hline Yes & 70 & & & \\
\hline Histology & & 0.044 & 0.025 & 0.043 \\
\hline Squamous cell carcinoma & 98 & & & \\
\hline Adenocarcinoma & 22 & & & \\
\hline Others ${ }^{a)}$ & 15 & & & \\
\hline RM (PRM + VRM) & & 0.203 & 0.026 & 0.000 \\
\hline Free of tumor & 122 & & & \\
\hline Positive or close ${ }^{b)}$ & 13 & & & \\
\hline PRM & & 0.015 & 0.000 & 0.000 \\
\hline Free of tumor & 128 & & & \\
\hline Positive or close $e^{c)}$ & 7 & & & \\
\hline VRM & & 0.453 & 0.822 & 0.465 \\
\hline Free of tumor & 128 & & & \\
\hline Positive or close & 7 & & & \\
\hline LN metastasis & & 0.292 & 0.000 & 0.000 \\
\hline No & 110 & & & \\
\hline Yes & 25 & & & \\
\hline Direct extension of parametrium & & 0.014 & 0.005 & 0.032 \\
\hline No & 107 & & & \\
\hline Yes & 28 & & & \\
\hline Para-aortic LN radiotherapy & & 0.649 & 0.849 & 0.178 \\
\hline No & 127 & & & \\
\hline Yes & 8 & & & \\
\hline Chemotherapy & & 0.703 & 0.006 & 0.038 \\
\hline No & 62 & & & \\
\hline Yes & 73 & & & \\
\hline
\end{tabular}

LRR, locoregional recurrence; DM, distant metastasis; OS, overall survival; RM, resection margin; PRM, parametrial resection margin; VRM, vaginal resection margin; LN, lymph node; FIGO, International Federation of Gynecology and Obstetrics.

${ }^{a)}$ Others were adenosquamous cell carcinoma $(n=12)$, malignant neuroendocrine tumor $(n=2)$, and carcinosarcoma $(n=1) .{ }^{b}$ Positive or close RM is composed of 6 patients with positive or close PRM only, 6 patients with positive or close VRM only, and one patient with both positive or close PRM or VRM. ${ }^{c}$ All patients with positive or close PRMs had direct extensions of parametrium (plIB). during each follow-up. Pap's smear was performed every 6 months. Radiographic examinations including chest X-ray, abdominopelvic CT or MRI were conducted every year.

Clinical data regarding treatment-related toxicities were collected. Toxicities that occurred more than 90 days after the start of radiotherapy were considered as late toxicities. Late toxicities were scored according to RTOG/EORTC late radiation morbidity scoring schema.

\section{Study end points and statistical analyses}

This study was designed to identify risk factors associated with local recurrence, regional recurrence, distant metastasis (DM), and overall survival (OS). Local recurrence was defined by either pathologic proof or an imaging study showing regrowth of tumor on the tumor bed or vaginal stump. Regional recurrence was defined by a new finding of metastatic pelvic LNs or organs below the aortic bifurcation. DM was defined as disease relapse outside the pelvis. The time to recurrence was defined from the date of diagnosis to the date of first recurrence.

Statistical analysis was performed using the Statistical Package for Social Sciences (SPSS) ver. 18 for Windows (SPSS Inc., Chicago, IL, USA). Kaplan-Meier method and the log-rank test were used to calculate the survival rate and differences according to prognostic factors, respectively. Multivariate analysis of prognostic factors was made by the Cox proportional hazards regression model. A p-value $<0.05$ was considered statistically significant.

\section{Results}

The median follow-up time was 60.0 months (range, 13 to 167 months). We excluded 6 patients because of short follow-up duration (under 12 month). One case with RT under 45.0 Gy was also excluded. After exclusion, 135 patients underwent retrospective evaluation of prognosis after being treated with PORT.

\section{Survival and failure pattern}

The 5-year progression-free survival and OS were 79\% and 91\%, respectively. The 5-year OS of patients with positive or close PRM was 19\% (Fig. 1). Treatment failure occurred in 29 patients (21\%) in the following groups: 1, 2, 1, 6, and 19 cases of local recurrence along with $(n=1)$, local recurrence along with DM $(n=2)$, regional recurrence alone $(n=1)$, regional recurrence along with DM ( $n=6)$, and DM alone $(n=19)$, respectively. Metastases were found in lung $(n=13)$, para- 


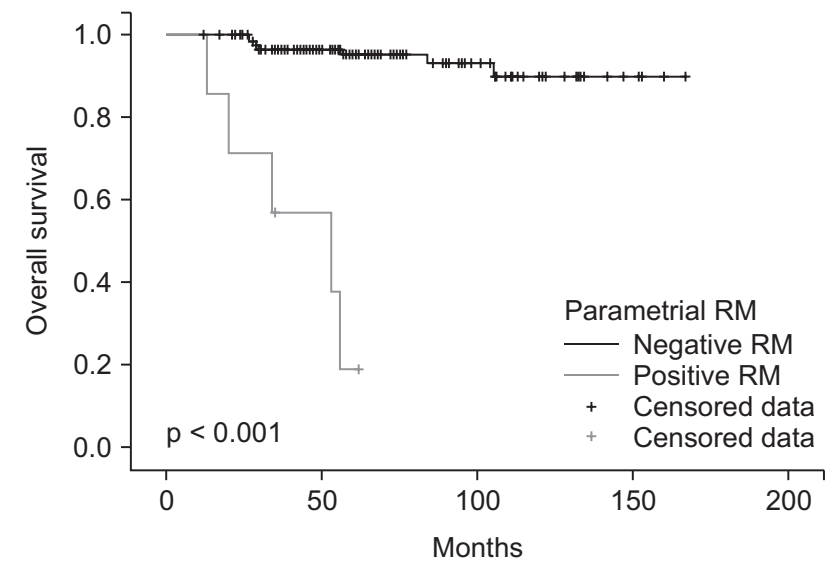

Fig. 1. Overall survival for 7 patients with positive or close parametrial resection margin (RM) and 128 patients without positive or close parametrial RM.

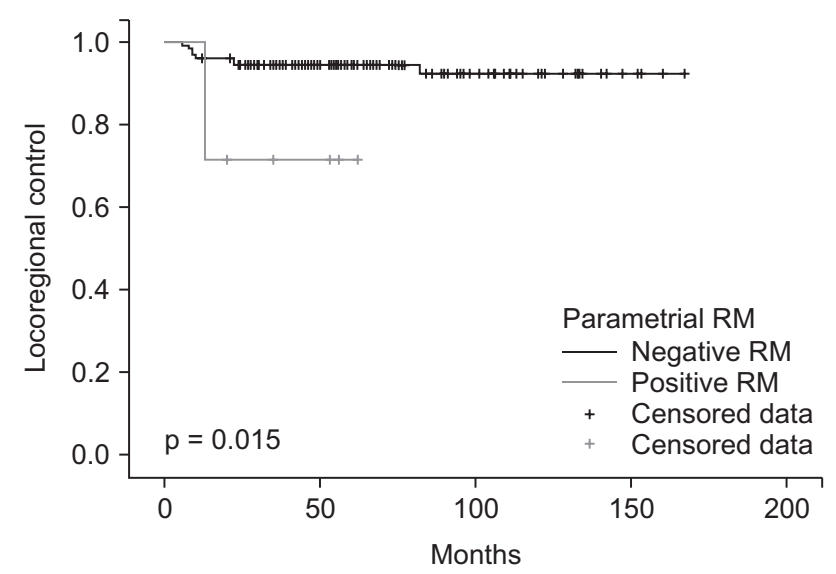

Fig. 2. Locoregional control for 7 patients with positive or close parametrial resection margin (RM) and 128 patients without positive or close parametrial RM.

aortic $L N(n=6)$, and peritoneum $(n=6)$.

\section{Clinical and pathologic risk factors predicting survival}

The risk factors for prognosis were evaluated by univariate and multivariate analyses. In univariate analysis, Iymphovascular invasion ( $p=0.029)$, histology of adenocarcinoma $(p=0.044)$, positive or close PRM ( $p=0.015)$, and direct extension of parametrium ( $p=0.014)$ were related with higher locoregional recurrence (LRR) (Fig. 2). Lymphovascular invasion ( $<<0.001)$, histology of adenocarcinoma ( $p=0.025)$, positive or close PRM ( $p<0.001)$, LN metastasis ( $p<0.001)$, direct extension of parametrium $(p=0.005)$, and chemotherapy $(p=0.006)$ were related with higher DM (Fig. 3).

Lymphovascular invasion ( $p=0.005)$, histology of adenocarcinoma ( $p=0.043)$, positive or close PRM $(p<0.001)$,

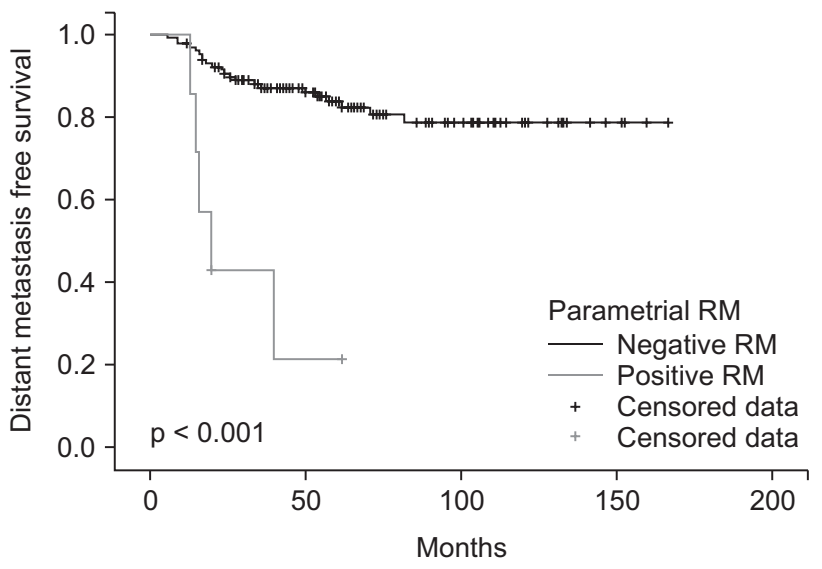

Fig. 3. Distant metastasis free survival for 7 patients with positive or close parametrial resection margin (RM) and 128 patients without positive or close parametrial RM.

LN metastasis $(p<0.001)$, direct extension of parametrium ( $p$ $=0.032)$, and chemotherapy $(p=0.038)$ were associated with poor OS (Table 1).

After multivariate analysis, the risk factors for DM were positive or close PRM ( $p=0.004)$, LN metastasis $(p=0.022)$, Iymphovascular invasion ( $p=0.044)$, and histology of adenocarcinoma ( $p=0.008)$. Positive or close PRM $(p<0.001)$ and LN metastasis $(p=0.009)$ were associated with OS (Table 2 ).

Grade 3 intestinal late toxicities were reported in 3 patients $(2 \%)$. No grade 4 late toxicity was observed.

\section{Discussion and Conclusion}

The treatment of uterine cervical cancer has developed as new risk factors have been discovered. During the era of surgical treatment alone, prognostic factors of cervical cancer included the following: margin status, number of positive LNs, extension of parametrium, depth of stromal invasion, tumor size, lymphovascular invasion, clinical stage, and patient age [6-14]. According to these studies, PORT was performed for patients with the aforementioned risk factors, especially for patients with positive pelvic LNs. The GOG 92 clinical trial compared RT after surgery with surgery alone in an intermediate-risk group. There was a reduction in the recurrence rate from $28 \%$ to $15 \%$ with the addition of RT [2].

However, even with PORT, OS of patients at high risk of relapse has remained a challenge to overcome with 50\% to $70 \%$ of patients falling into this category $[6-11,13]$. In a prospective randomized trial, combined chemotherapy with PORT for uterine cervical cancer patients with positive pelvic LNs, positive RM, and direct extension of parametrium, 
Table 2. Multivariate analysis of LRR, DM, and OS using the Cox proportional hazard model

\begin{tabular}{|c|c|c|c|c|c|}
\hline Prognosis & Clinicopathological factor & p-value & Wald & $\mathrm{RR}$ & $95 \% \mathrm{Cl}$ \\
\hline \multirow[t]{2}{*}{ LRR } & Direct extension of parametrium & 0.081 & 3.042 & 3.3 & $0.862-12.714$ \\
\hline & Lymphovascular invasion & 0.130 & 2.292 & 5.2 & $0.617-43.279$ \\
\hline \multirow[t]{6}{*}{ DM } & Positive or close PRM ${ }^{\text {a) }}$ & 0.004 & 4.839 & 3.7 & $1.154-12.071$ \\
\hline & LN metastasis & 0.022 & 5.584 & 3.0 & $1.208-7.601$ \\
\hline & Lymphovascular invasion & 0.044 & 4.062 & 3.7 & $1.037-13.121$ \\
\hline & Histology (comparing to SqCC) & 0.008 & - & - & - \\
\hline & Adenocarcinoma & 0.001 & 10.596 & 5.4 & $1.951-14.767$ \\
\hline & Others & 0.179 & 1.810 & 3.0 & $0.609-14.423$ \\
\hline \multirow[t]{2}{*}{ OS } & Positive or close PRM ${ }^{a)}$ & 0.000 & 18.899 & 19.3 & $5.085-73.449$ \\
\hline & LN metastasis & 0.009 & 6.843 & 8.2 & $1.693-39.293$ \\
\hline
\end{tabular}

None of the factors remained independent prognostic factors for LRR in cervical cancer after multivariate Cox regression, done by stepwise backward elimination according to Wald criteria. Redundant factors were positive of close PRMs and histology for LRR; direct extension of parametrium and chemotherapy for DM; and direct extension of parametrium, lymphovascular invasion, and chemotherapy for OS.

LRR, locoregional risk; $\mathrm{DM}$, distant metastasis; $\mathrm{OS}$, overall survival; $\mathrm{RR}$, relative risk; $\mathrm{Cl}$, confidence interval; PRM, parametrial resection margin; LN, lymph node; SqCC, squamous cell carcinoma.

${ }^{a}$ All patients with positive or close PRMs had direct extensions of parametrium (pllB).

resulted in improved OS [3]. The GOG 109 trial demonstrated that the addition of concurrent cisplatin-based chemotherapy to RT significantly improved PFS and OS for high-risk, earlystage patients, with a 4-year OS rate of 81\% [3]. In this trial, RT dose was 49.3 Gy to whole pelvis and vaginal brachytherapy was not permitted.

In our study, we analyzed prognostic factors for uterine cervical cancer patients who had undergone radical hysterectomy with adjuvant RT and concurrent chemotherapy on the basis of each patient's risk factors. Positive pelvic LNs and positive surgical margin remained statistically significant poor prognostic factors.

LN metastasis is one of the strongest prognostic factors. It elevates the possibility of DM so that systemic therapy such as chemotherapy will be a reasonable choice as an effort for improving prognosis. In addition, as a metastatic LN is still a poor prognostic factor even with cisplatinbased chemotherapy, further development of chemotherapy is needed to improve prognosis. However, unlike dissected metastatic LNs after surgical treatment, a positive RM stands for still remaining microscopic tumor cells in the operation bed. Therefore systemic chemotherapy combined with additional treatment to improve local control needs to be considered.

The survival rate of patients with positive RMs who have undergone PORT varies from 50\% to 80\% [12-18] (Table 3). Both definitive RT and surgery have been considered appropriate for patients with FIGO stage IA2, IB, and IIA carcinoma of the cervix $[7,8]$, and 5 -year OS of definitive RT for FIGO stage IB and IIA cervical cancer is 70\%-85\% $[8,19,20]$. According to the NCCN guidelines, if a patient has positive or close VRM after surgical treatment, additional vaginal cuff brachytherapy should be considered. However there is no exact recommendation regarding boosting for positive PRM [5].

In this study, vaginal brachytherapy was administered for the majority of patients with positive or close VRMs (5 patients out of 7). Vaginal brachytherapy dose for positive VRM was 3-4 Gy in 5 to 6 fractions, and total dose including EBRT dose was $65.4 \mathrm{~Gy}$ to $76.2 \mathrm{~Gy}$. The treatment result of patients who had positive or close VRMs has not shown inferior outcomes in terms of local control, DM, or OS compared to patients who had negative VRMs. This suggests that vaginal brachytherapy might have contributed to that result. On the other hand, only 2 patients out of 7 who had positive or close PRMs were treated with vaginal brachytherapy. Compared with previous studies, that is a very poor prognosis, even with adjuvant EBRT and chemotherapy [12-18]. This difference may be due to the different definition of close RM, as many studies defined close RM as within $5 \mathrm{~mm}$ while the current study defined it as within $1 \mathrm{~mm}$. Additionally, there are few publications examining PRM separately from VRM. Different anatomical locations have different lymphovascular systems, which may contribute to the different observed outcomes. In the current study, when whole positive or close RMs were considered (PRM and VRM), 5-year OS was 49\%, which is similar to other published studies. According to these data, addition of 


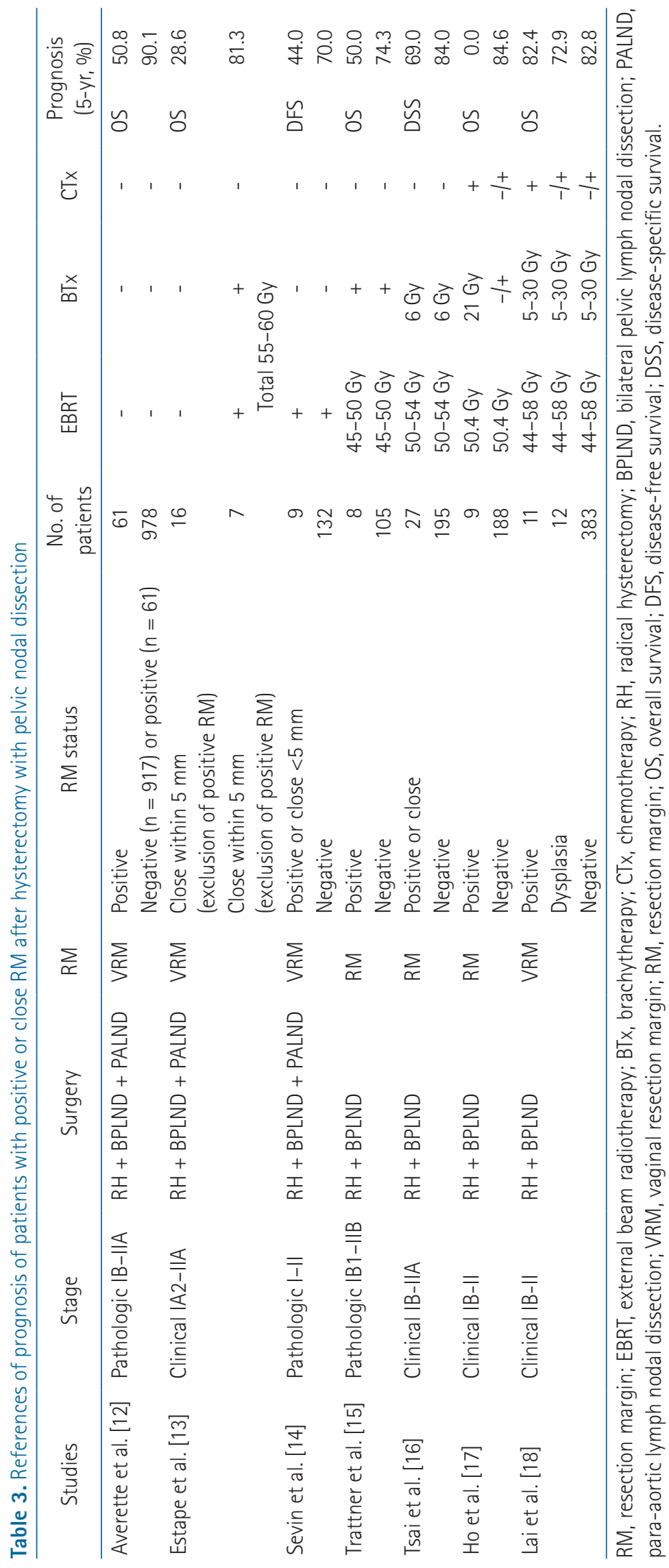


chemotherapy for patients with positive RM did not improve prognosis, especially for patients with positive PRMs. However, the small sample size of patients with positive or close PRM may have affected this conclusion.

Determining the sufficient threshold radiation dose for positive $\mathrm{RM}$ is an increasingly relevant issue. One study suggested that FIGO stage IA uterine cervical cancer can be treated with brachytherapy alone with LDR 65-75 Gy or HDR 7 Gy in 5-6 fractions (BED 59.5 to $71.4 \mathrm{~Gy}_{10}$ ) to achieve high local control rate. This result may be indirect evidence that conventional 50.4 Gy (BED $60.5 \mathrm{~Gy}_{10}$ ) to the true pelvis might be a sufficient dose even for RM-positive cancers. However, the question still remains whether this approach of comparing FIGO stage IA cancers with more advanced cancers with surgically positive RMs is reasonable or not. As a cancer progresses, new cancer clones develop and may contribute to RT resistance [21]. Therefore, even though FIGO stage IA cancers without surgery and more advanced cancers with surgically positive RMs have similar tumor burden quantity, tumor burden quality between them can be different. Additionally, anatomic location of the primary tumor has a significant effect on metastatic probability. Cervical cancer with direct extension of parametrium is considered to be locally advanced cancer; regardless of previous surgery based on clinical staging, if a patient has positive PRMs then PORT with a similar dosage of definitive RT is justified. The minimum definitive RT dose for cervical cancer is 70 Gy [19]. The NCCN guidelines recommend highly conformal boosts of an additional 10-15 Gy for limited volumes of gross unresected metastatic LNs. In this case, accumulated total dose to gross metastatic LNs is 60 Gy to 75 Gy [5]. Accordingly, a total dose above 60 Gy for positive PRM might be acceptable.

A positive PRM is an uncommon result for surgeons, and the numbers of patients with positive RMs are too few to conduct randomized trials to test an additional boosting dose; however, a comparison of prognosis may be possible after factoring for additional local control improvement.

In terms of histology, even though some studies failed to achieve statistical significance, adenocarcinoma has consistently been associated with a poorer prognosis than squamous cell carcinoma $[3,16,19]$. In this study, adenocarcinoma was associated with worse prognosis than squamous cell carcinoma, with a trend toward increased $\operatorname{LRR}(p=0.067)$ and a significantly increased rate of DM ( $p=0.037)$.

Depth of invasion is often mentioned as a major prognostic factor. According to the Sedlis criteria, depth of invasion, tumor size, and lymphovascular invasion should be considered when deciding on an adjuvant $\mathrm{RT}$ regimen $[2,22]$. However in this study depth of invasion was not significantly associated with prognosis, although there was a trend for association with OS ( $p=0.075)$. In multivariate analysis Iymphovascular invasion was correlated with DM ( $p=0.044)$.

The restrictions of this study include a relatively small sample size with only 13 of 135 patients of positive RMs and heterogeneous chemotherapy regimens. Although additional vaginal brachytherapy for positive or close VRM is an unswerving principle in our institution, 2 of 7 patients who had positive or close VRMs missed brachytherapy. On the other hand, 2 of 7 patients with positive or close PRMs treated with brachytherapy; one patient had both positive VRM and positive PRM and another patient treated with brachytherapy according to the responsible oncologist's decision. Nonhomogeneous groups of treatment made it difficult to draw an obvious conclusion from the result. Even though surgical staging system has been advocated by some clinicians, clinical staging more easily permits comparison of treatment results. In this study, we applied clinical staging system for prognostic factor analysis.

For the treatment of uterine cervical cancer, PORT following surgery in uterine cervical cancer showed excellent locoregional control and was associated with improved OS in this study. If PRM is positive or close after radical hysterectomy, CCRT combined with additional radiation boosting to increase the total dose to more than 50 Gy needs to be considered to improve local control rates.

\section{Conflict of Interest}

No potential conflict of interest relevant to this article was reported.

\section{References}

1. Waggoner SE. Cervical cancer. Lancet 2003;361:2217-25.

2. Rotman M, Sedlis A, Piedmonte $M R$, et al. A phase III randomized trial of postoperative pelvic irradiation in Stage IB cervical carcinoma with poor prognostic features: follow-up of a gynecologic oncology group study. Int J Radiat Oncol Biol Phys 2006;65:169-76.

3. Peters WA 3rd, Liu PY, Barrett RJ 2nd, et al. Concurrent chemotherapy and pelvic radiation therapy compared with pelvic radiation therapy alone as adjuvant therapy after radical surgery in high-risk early-stage cancer of the cervix. J Clin 
Oncol 2000;18:1606-13.

4. Rose PG. Chemoradiotherapy for cervical cancer. Eur J Cancer 2002;38:270-8.

5. National Comprehensive Cancer Network. NCCN Clinical Practice Guidelines in Oncology (NCCN Guidelines) [Internet]. Fort Washington, PA: National Comprehensive Cancer Network; c2015 [cited 2015 Jun 1]. http://www.nccn.org/professionals/ physician_gls/f_guidelines.asp.

6. Delgado G, Bundy B, Zaino R, Sevin BU, Creasman WT, Major F. Prospective surgical-pathological study of disease-free interval in patients with stage IB squamous cell carcinoma of the cervix: a Gynecologic Oncology Group study. Gynecol Oncol 1990;38:352-7.

7. Morley GW, Seski JC. Radical pelvic surgery versus radiation therapy for stage I carcinoma of the cervix (exclusive of microinvasion). Am J Obstet Gynecol 1976;126:785-98.

8. Hopkins MP, Morley GW. Radical hysterectomy versus radiation therapy for stage IB squamous cell cancer of the cervix. Cancer 1991;68:272-7.

9. Fuller AF Jr, Elliott N, Kosloff C, Hoskins WJ, Lewis JL Jr. Determinants of increased risk for recurrence in patients undergoing radical hysterectomy for stage IB and IIA carcinoma of the cervix. Gynecol Oncol 1989;33:34-9.

10. Inoue $T$, Okumura M. Prognostic significance of parametrial extension in patients with cervical carcinoma Stages IB, IIA, and IIB: a study of 628 cases treated by radical hysterectomy and lymphadenectomy with or without postoperative irradiation. Cancer 1984;54:1714-9.

11. Zreik TG, Chambers JT, Chambers SK. Parametrial involvement, regardless of nodal status: a poor prognostic factor for cervical cancer. Obstet Gynecol 1996;87(5 Pt 1):741-6.

12. Averette $H E$, Nguyen $H N$, Donato DM, et al. Radical hysterectomy for invasive cervical cancer: a 25-year prospective experience with the Miami technique. Cancer 1993;71(4 Suppl):1422-37.

13. Estape $R E$, Angioli $R$, Madrigal $M$, et al. Close vaginal margins as a prognostic factor after radical hysterectomy. Gynecol
Oncol 1998;68:229-32.

14. Sevin BU, Lu Y, Bloch DA, Nadji M, Koechli OR, Averette HE. Surgically defined prognostic parameters in patients with early cervical carcinoma: a multivariate survival tree analysis. Cancer 1996;78:1438-46.

15. Trattner M, Graf AH, Lax S, et al. Prognostic factors in surgically treated stage IB-IIB cervical carcinomas with special emphasis on the importance of tumor volume. Gynecol Oncol 2001;82: 11-6.

16. Tsai $\mathrm{CS}$, Lai $\mathrm{CH}$, Wang $\mathrm{CC}$, et al. The prognostic factors for patients with early cervical cancer treated by radical hysterectomy and postoperative radiotherapy. Gynecol Oncol 1999;75:328-33.

17. Ho CM, Chien TY, Huang SH, Wu CJ, Shih BY, Chang SC. Multivariate analysis of the prognostic factors and outcomes in early cervical cancer patients undergoing radical hysterectomy. Gynecol Oncol 2004;93:458-64.

18. Lai $\mathrm{CH}$, Hong JH, Hsueh $\mathrm{S}$, et al. Preoperative prognostic variables and the impact of postoperative adjuvant therapy on the outcomes of stage IB or II cervical carcinoma patients with or without pelvic lymph node metastases: an analysis of 891 cases. Cancer 1999;85:1537-46.

19. Landoni F, Maneo A, Colombo A, et al. Randomised study of radical surgery versus radiotherapy for stage IB-IIA cervical cancer. Lancet 1997;350:535-40.

20. Perez CA, Camel HM, Kuske RR, et al. Radiation therapy alone in the treatment of carcinoma of the uterine cervix: a 20-year experience. Gynecol Oncol 1986;23:127-40.

21. Nowell PC. The clonal evolution of tumor cell populations. Science 1976;194:23-8.

22. Sedlis A, Bundy BN, Rotman MZ, Lentz SS, Muderspach $L I$, Zaino RJ. A randomized trial of pelvic radiation therapy versus no further therapy in selected patients with stage IB carcinoma of the cervix after radical hysterectomy and pelvic Iymphadenectomy: a Gynecologic Oncology Group Study. Gynecol Oncol 1999;73:177-83. 